\title{
PROPAGATION AND INTERACTION OF EXTREMELY SHORT ELECTROMAGNETIC PULSES IN NON-LINEAR MEDIA
}

\author{
A.I.Maimistov \\ Moscow Engineering Physics Institute, Kashirskoe sh. 31, Moscow, Russia, \\ e-mail: maimistov@pico.mephi.ru
}

\begin{abstract}
Propagation of the extremely short electromagnetic pulse in non-linear dielectric media without the slowly varying envelope approximation is discussed. The models under consideration take into account both resonant and not-resonant excitations of non-linear medium, and polarisation states of electromagnetic wave. Steady state solutions of the relevant equations are presented for certain of these models.
\end{abstract}

PACS number: 42.65.R 


\section{Introduction}

In the context of rapid progress in the generation of femtosecond pulses of the electromagnetic radiation, it is of interest to consider the theoretical models of the pulses propagation in non-linear dispersive medium. Indeed, derivation of the evaluation equations for electromagnetic radiation is often based on the approximation of slowly varying complex envelopes of the optical pulses. This approximation is employed widely in theoretical studies devoted to nonlinear coherent phenomena. However, it is of interest to find a method of describing extremely short pulse (ESP) evolution without using the slowly varying envelope approximation.

A natural foundation for all the theories is the Maxwell equations supplemented with equations governing the evolution of the polarisation or currents arising in a medium subject to electromagnetic radiation. Since it seldom occurs that exact analytical results can be obtained in such problems, various approximations simplifying the consideration and allowing analytical expressions to be derived are often employed.

The typical models of the non-linear medium are the ensembles of $N$-level atoms or anharmonic oscillators. So, we can discuss the different degrees of approximation in the theory of interaction and propagation of optical pulses in framework of the resonant and non-resonant representation for medium [1].

\section{Extremely short pulse propagation in res- onance medium}

Let the spectral width of ESP be far smaller than the resonance frequency. This resonant condition allows to omit all non-resonant levels and to consider only two levels. Thus, we obtain the approximation of the two-level atoms [2], which is very popular in the resonant and coherent optics. The wave equation in this case is complemented by the Bloch equations for two-level atom variables.

\subsection{Propagation of the scalar ESP}

In general, the theory of the interaction of radiation with an ensemble of two-level atoms is based on the Bloch equations for atoms and the Maxwell 
equations for the classical electromagnetic field. In an isotropic dielectric the set of Maxwell equations reduced to one equation for the electric field $\vec{E}=E \vec{l}$. For a plane wave with constant polarisation vector one can obtain the following system of total Maxwell-Bloch (MB) equations

$$
\begin{gathered}
\frac{\partial^{2} E}{\partial z^{2}}-\frac{1}{c^{2}} \frac{\partial^{2} E}{\partial t^{2}}=\frac{4 \pi n_{A} d}{c^{2}}\left\langle\frac{\partial^{2} r_{1}}{\partial t^{2}}\right\rangle \\
\frac{\partial r_{1}}{\partial t}=-\omega_{a} r_{2}, \quad \frac{\partial r_{2}}{\partial t}=\omega_{a} r_{1}+\frac{2 d}{\hbar} E r_{3}, \quad \frac{\partial r_{3}}{\partial t}=-\frac{2 d}{\hbar} E r_{2}
\end{gathered}
$$

where $d$ is the projection of a matrix element of the dipole operator on the direction of $\vec{l}, n_{A}$ is the concentration of resonant atoms. It should be noted that the components of Bloch vector $r_{1}, r_{2}$, and $r_{3}$ depend on the atomic resonance frequency $\omega_{a}$. Hereafter the angular brackets represent summation over all the atoms characterised by the frequency $\omega_{a}$.

Let us consider the solution of equations (1) and (2) describing a solitary steady-state wave. Boundary conditions at $|t| \rightarrow \infty$ must look like the following

$$
E=\partial E / \partial t=0, \quad r_{2}=r_{1}=0, \quad r_{3}=-1 .
$$

To obtain the expressions describing the propagation of stationary ESP one should suppose that the components of the Bloch vector and the electric field $E$ depend on only one variable $t \pm z / V$ or $\eta=\omega_{a}(t \pm z / V)$ in dimensionless form. That means that a stationary (steady state) wave propagates only in one (positive or negative) direction. Under this assumption the system (1), (2) transforms into the system of ordinary differential equations which can be solved by usual methods. Solution of the these ordinary differential equations leads to the expression for electric field strength

$$
E(z, t)=E_{0} \sec h\left[d E_{0}(t \pm z / V) / \hbar\right],
$$

where pulse amplitude is related with pulse duration $t_{p}$ as $E_{0}=\hbar t_{p}^{-1} d^{-1}$. This expression for stationary pulse correlates with the one found in [3, 4]. Expression for the stationary ESP velocity can be obtained from definitions of pulse duration and amplitude. As in self-induced theory by McCall-Hahn [2, 5], these values are related to each other, so the pulse is able to invert a two-level system and then return it to the initial state while the pulse lasts. After some algebra one may find

$$
\frac{1}{V^{2}}=\frac{1}{c^{2}}\left(1+\frac{8 \pi n_{A} d^{2} \hbar \omega_{a}}{\left(d E_{0}\right)^{2}+\left(\hbar \omega_{a}\right)^{2}}\right),
$$


that corresponds to what was found in [3].

\subsection{Propagation of the ESP of polarised radiation}

In this case generalised Maxwell-Bloch equations can be written as follows

$$
\begin{gathered}
\frac{\partial^{2} E^{(+1)}}{\partial z^{2}}-\frac{1}{c^{2}} \frac{\partial^{2} E^{(+1)}}{\partial t^{2}}=\frac{4 \pi n_{A}}{c^{2}} \frac{\partial^{2}}{\partial t^{2}}\left\langle d_{13} \rho_{31}+d_{31} \rho_{13}\right\rangle, \\
\frac{\partial^{2} E^{(-1)}}{\partial z^{2}}-\frac{1}{c^{2}} \frac{\partial^{2} E^{(-1)}}{\partial t^{2}}=\frac{4 \pi n_{A}}{c^{2}} \frac{\partial^{2}}{\partial t^{2}}\left\langle d_{23} \rho_{32}+d_{32} \rho_{23}\right\rangle, \\
i \hbar \frac{\partial \rho_{13}}{\partial t}=-\hbar \omega_{a} \rho_{13}+d_{13}\left(\rho_{33}-\rho_{11}\right) E^{(+1)}-d_{23} \rho_{12} E^{(-1)}, \\
i \hbar \frac{\partial \rho_{23}}{\partial t}=-\hbar \omega_{a} \rho_{23}+d_{23}\left(\rho_{33}-\rho_{22}\right) E^{(-1)}-d_{13} \rho_{21} E^{(+1)}, \\
i \hbar \frac{\partial \rho_{12}}{\partial t}=d_{13} \rho_{32} E^{(+1)}-d_{32} \rho_{13} E^{(-1)}, \\
i \hbar \frac{\partial}{\partial t}\left(\rho_{11}-\rho_{33}\right)=2\left(d_{13} \rho_{31}-d_{31} \rho_{13}\right) E^{(+1)}-\left(d_{23} \rho_{32}-d_{32} \rho_{23}\right) E^{(-1)}, \\
i \hbar \frac{\partial}{\partial t}\left(\rho_{22}-\rho_{33}\right)=\left(d_{13} \rho_{31}-d_{31} \rho_{13}\right) E^{(+1)}-2\left(d_{23} \rho_{32}-d_{32} \rho_{23}\right) E^{(-1)},
\end{gathered}
$$

where $E^{(q)}$ is the spherical $q$ component of the vector of electrical field strength of a light wave, $q= \pm 1, d_{k l}$ are the matrix elements of the dipole moment operator of the atomic transition. Hereafter $d_{13}=d_{23}=d_{31}^{*}=d_{32}^{*}$.

By supposing that the matrix elements of the density matrix $\rho_{k l}$, and the electric field $E$ depend on only one variable $t \pm z / V$ we can find the expression for the strength of the electrical field of the ESP in the form

$$
E^{( \pm 1)}(z, t)=e^{( \pm)} E_{0} \sec h\left[d E_{0}(t \pm z / V) / \hbar\right]
$$

where $e^{( \pm)}$are the components of a unit vector of electromagnetic fields. This is a simple generalisation of the results obtained by Bullough and Ahmad [4] to the case of vector (polarised) ESP and specific model of a resonant medium. As in Ref. [4], the pulse duration tp is expressed in terms of the pulse peak amplitude $t_{p}=\hbar\left(d E_{0}\right)^{-1}$. The propagation velocity of the ESP is:

$$
\frac{1}{V^{2}}=\frac{1}{c^{2}}\left(1+\frac{8 \pi n_{A} d^{2} \hbar \omega_{a}}{2\left(d E_{0}\right)^{2}+\left(\hbar \omega_{a}\right)^{2}}\right)
$$


Besides the solitary wave solutions, equations (14), (2) and (5)-(17) have the solutions in the form of cnoidal wave.

The above analysis of the ESP propagation shows that the nature of such stationary ESP depends on the state of the medium. The scalar solution [4] can be trivially generalised to cover a vector video pulse case under condition $n_{10}=n_{20}=1$. So we obtain a circular polarised pulse [6] with duration of a half of a reciprocal atomic transition frequency. The propagation velocity of this pulse does not depend on the conditions of its polarisation and the value of this velocity is in agreement with what was found in 4 .

The new solution of the generalised complete Maxwell-Bloch equations arises when initially the resonance medium has an asymmetrical distribution of level populations (i.e., $n_{10}$ and $n_{20}$ ) of the excited states that have different projections of the angular momentum: $n_{10} \neq n_{20}[7]$. One of the spherical components of the electric field strength vector behaves as in the scalar case, i.e., it is a unipolar spike of the electrical field. Another component is a variable-sign solitary wave. All stationary solutions like these form a threeparametric family, where each member is defined by the propagation speed $V$ and $n_{10}, n_{20}$ determining the polarisation state of the pulse.

\section{Extremely short pulse propagation under quasi-resonance condition}

Let assume that the amplitude of electrical field of electromagnetic pulse is so small that the instant Rabi frequency $\omega_{R}$ proves to be far less then the frequency of the resonance transition, i.e.,

$$
\varepsilon=\frac{\omega_{R}}{\omega_{a}}=\frac{\left|d_{13}\right| E_{0}}{\hbar \omega_{a}} \ll 1,
$$

where $\omega_{R}$ corresponds to the wave of constant electrical field amplitude $E_{0}=$ $\max \left|E^{(q)}\right|$. In this case the solution of Bloch equations ( 团) can be presented formally [8] by power series in $\varepsilon$. Limiting ourselves to the terms of this series up to $\varepsilon^{3}$, for polarisation in equations (5) and (6), $P^{(+1)}=d_{13} \rho_{31}+d_{31} \rho_{13}$, $P^{(-1)}=d_{23} \rho_{32}+d_{32} \rho_{23}$, we obtain expressions [8]:

$$
P^{(q)}=-\frac{2 \sigma\left|d_{13}\right|^{2}}{\hbar \omega_{a}}\left\{E^{(q)}-\frac{1}{\omega_{a}^{2}} \frac{\partial^{2} E^{(q)}}{\partial t^{2}}-\frac{2\left|d_{13}\right|^{2}}{\left(\hbar \omega_{a}\right)^{2}}(\vec{E} \cdot \vec{E}) E^{(q)}\right\} .
$$


The substitution of (11) into equations (51) and (6) results in the non-linear wave equation, which gives approximate description of the extremely-short pulse of electromagnetic wave

$$
\frac{\partial^{2} \vec{E}}{\partial z^{2}}-\frac{1}{V^{2}} \frac{\partial^{2} \vec{E}}{\partial t^{2}}-b \frac{\partial^{4} \vec{E}}{\partial t^{4}}-a \frac{\partial^{2}}{\partial t^{2}}\{(\vec{E} \cdot \vec{E}) \vec{E}\}=0
$$

where

$$
a=\left\langle\frac{16 \pi n_{A} \sigma\left|d_{13}\right|^{2}}{c^{2}\left(\hbar \omega_{a}\right)^{3}}\right\rangle, \quad b=\left\langle\frac{8 \pi n_{A} \sigma\left|d_{13}\right|^{2}}{c^{2} \hbar \omega_{a}^{3}}\right\rangle .
$$

The velocity of pulse propagation $V$ alters because of the dispersion, which is due to the resonant medium:

$$
V^{-2}=c^{-2}\left[1-\left\langle 8 \pi n_{A} \sigma\left|d_{13}\right|^{2} / \hbar \omega_{a}\right\rangle\right]
$$

For zero boundary conditions as $t \rightarrow \pm \infty$ the steady state solution of equation (12) can be written in the form:

$$
\vec{E}(z, t)=\vec{e} E_{0} \operatorname{sech}\left[\left(t \pm \alpha z / V-t_{0}\right) / t_{S}\right]
$$

where

$$
t_{S}=E_{0}^{-1} \sqrt{|b| / 2|a|}, \quad \alpha=\sqrt{1+2 \sigma E_{0}^{2} V^{2}|a|} .
$$

Thus, the steady state solutions of the equation (12) describe the extremely short pulse propagation with constant polarisation state (i.e., $\vec{e}=$ const ) of this electromagnetic wave.

Spectral half-width of the ESP (13) is $t_{S}^{-1}$. Consequently the resonance condition will be applicable if $t_{S}^{-1} \ll \bar{\omega}$. From the definition of the parameters $|a|$ and $|b|$ we can estimate their ratio as $|a| /|b| \approx 2\left|d_{13}\right|^{2} \bar{\omega}^{2} \hbar^{-2}$ (where we neglected inhomogeneous broadening and all the frequencies $\omega_{a}$ were replaced by $\bar{\omega})$. Thus, the resonance condition complies with the condition (10) $\omega_{R} \ll$ $\bar{\omega}$.

Besides the solitary steady state wave (13) both dark solitary waves and a periodic stationary waves can be found. However they contradict with conditions of applicability of the initial equations for atomic variables, in where any relaxation processes are not taking into account. The considering pulse is short one in this sense. 


\section{Extremely short pulse propagation in non- resonance medium}

As it was pointed in [9] when the ESP field approaches the atomic field $\left(\sim 10^{8}\right.$ - $10^{9} \mathrm{~V} / \mathrm{cm}$ ), ESP formation is affected mainly by the atomic ionisation potential, which limits pulse duration and amplitude. For such fields the resonant models are invalid. However, one can evaluate limitations on ESP within a classical model of an atom with a strong non-linear potential, limited at infinity, to allow for ionisation. Then the (generalised) Bloch equations are replaced by classical equations for the electron (and ion) motion.

\subsection{Scalar Duffing model}

Let the medium be a non-linear and non-resonant one. A similar approach was exploited [10] to develop the self-induced transparency in ionic crystals in the framework of the Duffing's type model. The scalar wave equation is

$$
\frac{\partial^{2} E}{\partial z^{2}}-\frac{1}{c^{2}} \frac{\partial^{2} E}{\partial t^{2}}=\frac{4 \pi}{c^{2}} \frac{\partial^{2} P}{\partial t^{2}} .
$$

We complete this wave equation by the non-linear oscillator equation for the medium. If $X$ is the displacement of an electron from its equilibrium position, the motion equation (which neglects friction) can be written as

$$
\frac{\partial^{2} X}{\partial t^{2}}+\omega_{0}^{2} X+\kappa_{3} X^{3}=\mathfrak{L} \frac{e}{m} E
$$

where $\omega_{0}$ is an eigenfrequency of the oscillator, while $\kappa_{3}$ is anharmonicity coefficient. The term on the right-hand side of equation (15) represents the force exerted on the electron by the electromagnetic field, where $\mathfrak{L}=(\varepsilon+2) / 3$ is the Lorentz factor, $e$ is the electron's electric charge, and $m$ is its mass. However, it is possible to absorb $\mathfrak{L}$ into an effective mass $m_{\text {eff }}=m / \mathfrak{L}$. Hereafter, we will use $m$ as a symbol for this effective mass. Finally, the dynamical variable $X$ is related to the medium's polarisation, $P=n_{A} e X$, where $n_{A}$ is the density of the oscillators (atoms).

The steady state solutions of the system of equations (14) and (15) can be found if we suppose that the displacement $X$ and electric strength $E$ depend on one variable $\eta=\omega_{0}(t \pm z / V)$. Here velocity $V$ is a parameter of the 
solution. Thus, the expression for electric field of ESP can be obtained by solving the ordinary non-linear differential equations. That leads to

$$
E(t \pm z / V)=E_{m} \alpha(\alpha-1)^{1 / 2} \operatorname{sech}\left[(\alpha-1)^{1 / 2} \omega_{0}\left(t \pm z / V-t_{0}\right)\right]
$$

where $E_{m}=\left(m \omega_{0}^{3} / e\right) \sqrt{2 / \kappa_{3}}, \alpha=\left(\omega_{p} / \omega_{0}\right)^{2} V^{2} /\left(c^{2}-V^{2}\right)$, and $\omega_{p}=\left(4 \pi n_{A} e^{2} / m\right)^{1 / 2}$ is the plasma frequency:

\subsection{Vector Duffing model}

Let us consider the ESP propagation in a non-linear medium, which is again presented by an ensemble of the molecules. However, now let us suppose that the inner degrees of freedom are described by the potential:

$$
U(X, Y)=\frac{1}{2} \omega_{1} X^{2}+\frac{1}{2} \omega_{2} Y^{2}+\frac{1}{2} \kappa_{2} X^{2} Y^{2}+\frac{1}{4} \kappa_{4}\left(X^{4}+Y^{4}\right)
$$

where $\kappa_{2}$ and $\kappa_{4}$ are anharmonicity coefficients. If the coupling parameter $\kappa_{2}=0$, then this potential describes the scalar Duffing model of anharmonic oscillator, which can oscillate independently in two orthogonal directions. Expression (17) is the simplest generalisation of this model. Polarisation of the molecule is defined by the expression $\vec{p}=e X \vec{e}_{1}+e Y \vec{e}_{2}$, and the total polarisation $\vec{P}$ is the product of the density $n_{A}$ and the polarisation of one molecule.

The propagation of the ESP will be considered in the unidirectional wave approximation, so the wave equations for electric field corresponding to the different polarisation components of the ESP can be written as follows

$$
\frac{\partial^{2} E_{1}}{\partial z^{2}}-\frac{1}{c^{2}} \frac{\partial^{2} E_{1}}{\partial t^{2}}=\frac{4 \pi n_{A} e}{c^{2}} \frac{\partial^{2} X}{\partial t^{2}}, \quad \frac{\partial^{2} E_{2}}{\partial z^{2}}-\frac{1}{c^{2}} \frac{\partial^{2} E_{2}}{\partial t^{2}}=\frac{4 \pi n_{A} e}{c^{2}} \frac{\partial^{2} Y}{\partial t^{2}} .
$$

The equations of motion for anharmonic oscillator follow from the classical Newton equations

$$
\begin{aligned}
& \frac{\partial^{2} X}{\partial t^{2}}+\omega_{1}^{2} X+\kappa_{2} X Y^{2}+\kappa_{4} X^{3}=\frac{e}{m} E_{1} \\
& \frac{\partial^{2} Y}{\partial t^{2}}+\omega_{2}^{2} Y+\kappa_{2} Y X^{2}+\kappa_{4} Y^{3}=\frac{e}{m} E_{2} .
\end{aligned}
$$

It is possible to find a steady state solution of this system of equations. Let us suppose the fields depend only on $\tau=t \pm z / V$. Integrating equations 
(18) and taking account of the boundary conditions, which correspond to the vanishing electric field of the USP and polarisation of molecules at infinity, we find that $E_{1}=4 \pi n_{A} e \theta X$ and $E_{2}=4 \pi n_{A} e \theta Y$. Substitution of this result into (19) and (20) leads to the following equations

$$
\begin{aligned}
& \frac{d^{2} X}{d \tau^{2}}+\left(\kappa_{2} Y^{2}+\kappa_{4} X^{2}\right) X=\left(\theta \omega_{p}^{2}-\omega_{1}^{2}\right) X \\
& \frac{d^{2} Y}{d \tau^{2}}+\left(\kappa_{2} X^{2}+\kappa_{4} Y^{2}\right) Y=\left(\theta \omega_{p}^{2}-\omega_{2}^{2}\right) X
\end{aligned}
$$

where $\theta=V^{2}\left(c^{2}-V^{2}\right)^{-1}$ These equations appear in many works, so we can easily find their solutions. Let suppose that the eigenfrequencies $\omega_{1}$ and $\omega_{2}$ are equal. The partial solution of system (21), (22) in this case is

$$
E_{1}(\tau)=E_{2}(\tau)=E_{m} \alpha(\alpha-1)^{1 / 2} \operatorname{sech}\left[(\alpha-1)^{1 / 2} \omega_{1}\left(\tau-\tau_{0}\right)\right],
$$

where $E_{m}=\left(m \omega_{1}^{3} / e\right) \sqrt{2 /\left(\kappa_{2}+\kappa_{4}\right)}, \tau_{0}$ is the integration constant. This solution similar to that above describes the linear polarised video pulse propagating without distortion in the considered non-linear medium.

The more interesting solution exists if the frequencies $\omega_{1}$ and $\omega_{2}$ are different, but factors of anharmonicity coincide $\kappa_{2}=\kappa_{4}$. In this case the solution of the system of equations (21) and (22) leads to following expressions [11:

$$
\begin{aligned}
& E_{1}(\tau)=\frac{8 \pi e n_{A} \theta \sqrt{2 \kappa_{2}^{-1}} \mu_{1} \exp \left(\theta_{1}\right)\left\{1+\exp \left(2 \theta_{2}+\mu_{12}\right)\right\}}{1+\exp \left(2 \theta_{1}\right)+\exp \left(2 \theta_{2}\right)+\exp \left(2 \theta_{1}+2 \theta_{2}+\mu_{12}\right)}, \\
& E_{2}(\tau)=\frac{8 \pi e n_{A} \theta \sqrt{2 \kappa_{2}^{-1}} \mu_{2} \exp \left(\theta_{2}\right)\left\{1+\exp \left(2 \theta_{1}+\mu_{12}\right)\right\}}{1+\exp \left(2 \theta_{1}\right)+\exp \left(2 \theta_{2}\right)+\exp \left(2 \theta_{1}+2 \theta_{2}+\mu_{12}\right)},
\end{aligned}
$$

where

$$
\exp \mu_{12}=\left(\mu_{1}-\mu_{2}\right)\left(\mu_{1}+\mu_{2}\right)^{-1} \text {. }
$$

In these expressions the following parameters $\mu_{1,2}=\left(\theta \omega_{p}^{2}-\omega_{1,2}^{2}\right)$ and $\theta_{1,2}=$ $\mu_{1,2}\left(\tau-\tau_{1,2}\right)$ are used, where $\tau_{1,2}$ are the integration constants. Solutions (23) describe the steady state propagation of the ESP where one of these components corresponds to a unipolar spike of the electric field, sub-cycle pulse. The other component is a bipolar impulse or monocycle pulse. 


\subsection{Cubic-quadratic Duffing model}

An objective of the present section is to discuss the propagation and interactions of linearly polarised USPs in a non-linear dispersive medium modelled by an anharmonic oscillator combining quadratic and cubic nonlinearities. As is well known, in the case when the oscillator is quasi-harmonic, the quadratic and cubic non-linear terms produce effects of the same order of magnitude [12], that is why it is natural to consider a mixed model of this type. It is also relevant to mention that modelling dynamics of the broad (rather than ultra-short) optical solitons in a medium with competing quadratic and $\mathrm{cu}-$ bic nonlinearities has recently attracted considerable attention, see, e.g., the works 13 and references therein.

The one-dimensional propagation of the electromagnetic waves in a nonlinear medium is governed by the wave equation (14). We adopt a simple anharmonic oscillator model for the medium, which is a frequently used approximation [14] (see also [15, 16]). If $X$ is the displacement of an electron from its equilibrium position, the motion equation (which neglects friction) can be written as

$$
\frac{\partial^{2} X}{\partial t^{2}}+\omega_{0}^{2} X-\kappa_{2} X^{2}+\kappa_{3} X^{3}=\frac{e}{m} E
$$

where $\kappa_{2}$ and $\kappa_{4}$ are anharmonicity coefficients. It seems plausible that the system of equations (14), (24) is not an integrable one. Nevertheless, some exact analytical solutions, describing the propagation of ESPs, can be found. To this end, one assumes that $E$ and $X$ depend on a single variable, $\eta=$ $\omega_{0}(t \pm z / V)$ with some velocity $V$. It is convenient to express the velocity $V$ of a steadily moving pulse by introducing the constant $\alpha$

$$
\alpha=\left(\frac{\omega_{p}}{\omega_{0}}\right)^{2} \frac{V^{2}}{c^{2}-V^{2}},
$$

where $\omega_{p}$ is the plasma frequency. The first equation of the system, i.e., (14), can be integrated. It allow us to obtain a family of exact solutions parameterised by the continuous positive parameter $(\alpha-1)$ and discrete one

$\sigma= \pm 1$,

$$
E^{(+)}(\eta ; \alpha)=\frac{3 E_{m} \alpha(\alpha-1)}{\sqrt{1+9(\alpha-1) \mu} \cosh (\sqrt{(\alpha-1)} \eta)-1}
$$




$$
E^{(-)}(\eta ; \alpha)=-\frac{3 E_{m} \alpha(\alpha-1)}{\sqrt{1+9(\alpha-1) \mu} \cosh (\sqrt{(\alpha-1)} \eta)+1},
$$

where $E_{m}=\left(m \omega_{0}^{4} / e\left|\kappa_{2}\right|\right), \mu=\left(\kappa_{3} \omega_{0}^{2} / 2 \kappa_{2}^{2}\right)$, and the superscript standing for $\sigma$. The pulses represented by the solutions (33) and (34) have different polarities. In the limit $\kappa_{3} \rightarrow 0$, corresponding to the model with a purely quadratic nonlinearity, equation (26) goes over into a singular solution,

$$
E^{(+)}(\eta ; \alpha)=\frac{3 E_{m} \alpha(\alpha-1)}{2 \sinh ^{2}(\sqrt{(\alpha-1)} \eta / 2)},
$$

while expression (27) yields a non-singular pulse in the same limit, which was already found in Ref. [17,

$$
E^{(-)}(\eta ; \alpha)=-\frac{3 E_{m} \alpha(\alpha-1)}{2 \cosh ^{2}(\sqrt{(\alpha-1)} \eta / 2)} .
$$

Thus, in compliance with the results of Ref. [17], only one family of nonsingular pulses exists in the case of the purely quadratic nonlinearity. We stress that the velocities of two pulses, which have the opposite polarities but the same value of $\alpha$ are equal, since they are determined by equation (25) and depend only on $\alpha$.

Although the pulse solutions have been obtained in the exact form, their stability and interactions should be studied by means of numerical simulations. This investigation was performed under unidirectional approximation. Simulations of interactions between have shown that their collisions are quasi-elastic, irrespective of the polarities of the colliding pulses: after passing through each other, the pulses retrieve the same shapes and velocities as they had before the collision, the only result being a shift of their centers. However, the character of the interaction between pulses with equal and opposite polarities becomes different with the decrease of their relative velocity. In the case of equal polarities, the pulses with a small relative velocity demonstrate strong mutual repulsion: the distance between them attains a minimum, and then they start to separate again, so that they never completely overlap. Strong energy exchange between the pulses takes place around the point where they attain the minimum separation. The energy exchange gives rise to mutual interconversion of the two pulses, so that after the collision they, effectively, swap their positions. 


\subsection{Vector model of an quadratic non-linear media}

The propagation of an ESP of polarised radiation in a medium with a quadratic nonlinearity can be considered by using a model of an ensemble of two-component anharmonic oscillators [18]. This model described by the wave equations (18) supplemented with the following equations

$$
\begin{aligned}
& \frac{\partial^{2} X}{\partial t^{2}}+\omega_{1}^{2} X+\kappa_{111} X^{2}+2 \kappa_{112} X Y+\kappa_{122} Y^{2}=\frac{e}{m_{1}} E_{1} \\
& \frac{\partial^{2} Y}{\partial t^{2}}+\omega_{2}^{2} Y+\kappa_{112} X^{2}+2 \kappa_{122} X Y+\kappa_{222} Y^{2}=\frac{e}{m_{2}} E_{2}
\end{aligned}
$$

where the anharmonicity coefficients $\kappa_{i j l}$ and two effective masses $m_{1}$ and $m_{2}$ are introduced. Let the electric field strengths and displacements $X$ and $Y$ depend only on the variable $\tau=t \pm z / V$. Similar to the scalar case, one can integrate the wave equations (18) and equations (28), (29) can be reduced into the following set of non-linear equations

$$
\begin{aligned}
& \frac{d^{2} X}{d \tau^{2}}+\left(\omega_{1}^{2}-\theta \omega_{p}^{2}\right) X+\kappa_{111} X^{2}+2 \kappa_{112} X Y+\kappa_{122} Y^{2}=0 \\
& \frac{d^{2} Y}{d \tau^{2}}+\left(\omega_{2}^{2}-\theta \omega_{p}^{2}\right) Y+\kappa_{112} X^{2}+2 \kappa_{122} X Y+\kappa_{222} Y^{2}=0 .
\end{aligned}
$$

If both the eigenfrequencies of oscillators and the anharmonicity coefficients are equal to each other, then the problem considered can by reduced to the

scalar one. Unfortunately, the general solution of the equations (30), (31) is unknown.

\subsection{Coupled between vibrations and light waves in Ra- man media}

To description of the light propagation in Raman medium in [19] the oscillator model has been proposed. The displacement $Q$, vibration density $\rho$, and electric field of the light wave $E$ are governed by the following equations

$$
\begin{gathered}
\frac{\partial^{2} E}{\partial z^{2}}-\frac{1}{c^{2}} \frac{\partial^{2} E}{\partial t^{2}}=\frac{4 \pi}{c^{2}} \frac{\partial^{2} P}{\partial t^{2}} \\
\frac{\partial^{2} Q}{\partial t^{2}}+\frac{1}{T_{2}} \frac{\partial Q}{\partial t}+\omega_{0}^{2} Q=-\frac{\alpha_{1}}{2 M} E^{2} \rho
\end{gathered}
$$




$$
\frac{\partial \rho}{\partial t}+\frac{1}{T_{1}}\left(\rho-\rho_{0}\right)=-\frac{\alpha_{1}}{\hbar \omega_{0}} E^{2} \frac{\partial Q}{\partial t},
$$

where $\omega_{0}$ is an eigenfrequency of the oscillator, $M$ is a mass of ion. The nonlinear polarisation is defined by expression $P=n_{A} \alpha_{1} Q E$, where parameter $\alpha_{1}$ is the coefficient in expansion of the atomic (or molecular) polarizability $\alpha(Q) \approx \alpha_{0}+\alpha_{1} Q$. The relaxation processes were taken into account by introduction relaxation times $T_{1}$ and $T_{2}$. The constraint $\rho=$ const $=\rho_{0}=$ -1 leads this model to the Placzek one describing the stimulated Raman scattering [20]. The typical magnitude of the parameters are $\omega_{0}=10^{13} \mathrm{~s}^{-1}$, $M=10^{-22} \mathrm{~g}, \alpha_{1}=10^{-15} \mathrm{~cm}^{2}, n_{A}\left|\rho_{0}\right|=10^{22} \mathrm{~cm}^{-3}$.

The steady state solutions of the Blomberge-Shen model (32) are not known. It was shown that under unidirectional approximation (when wave propagates only in one of the possible directions) 21 this model can be used to description of the self-induced transparency under two-photon resonance.

\subsection{Isotropic solid dielectric model}

As pointed out in [22], in the study of the ESP propagation in a solid dielectric medium it is essential that the material equations adequately account for dispersion of both the linear and the non-linear susceptibility of the medium over practically its entire transparency range. Taking into account the contributions of both the electron and phonon subsystems of the solid dielectric one can describe normal and anomalous group dispersion. Thus, the simplest generation of the Lorentz model was proposed

$$
\frac{\partial^{2} P}{\partial t^{2}}+\left(\omega_{1}^{2}+\gamma E^{2}\right) P=n_{A} \alpha_{0} E+\beta R E, \quad \frac{\partial^{2} R}{\partial t^{2}}+\omega_{2}^{2} R=\chi P E,
$$

where $P$ is electronic polarisation, the extra variable $R$ reflects vibration motion of the atoms. Parameters $\alpha_{0}, \beta, \gamma, \chi$ are the characteristic ones to phenomenological description of the dielectric medium. The more complete model was proposed in [23]. The generalised Lorentz model represented there by the set of equations

$$
\begin{gathered}
\frac{\partial^{2} P_{e}}{\partial t^{2}}+\frac{2}{T_{e}} \frac{\partial P_{e}}{\partial t}+\omega_{e}^{2} P_{e}=\alpha_{e} E+\beta\left(R_{e}+R_{v}\right) E \\
\frac{\partial^{2} P_{i}}{\partial t^{2}}+\frac{2}{T_{i}} \frac{\partial P_{i}}{\partial t}+\omega_{i}^{2} P_{i}=\alpha_{i} E
\end{gathered}
$$




$$
\begin{gathered}
\frac{\partial^{2} R_{e}}{\partial t^{2}}+\frac{2}{T_{e 1}} \frac{\partial R_{e}}{\partial t}+\omega_{e 1}^{2} R_{e}=\gamma_{e}\left(P_{e}+P_{i}\right) E \\
\frac{\partial^{2} R_{v}}{\partial t^{2}}+\frac{2}{T_{v}} \frac{\partial R_{v}}{\partial t}+\omega_{v}^{2} R_{v}=\gamma_{v}\left(P_{e}+P_{i}\right) E
\end{gathered}
$$

where $P_{e}$ and $P_{i}$ are the contributions to the polarisation of the electronic and ionic components. The oscillators described by the variables $R_{e}$ and $R_{v}$ give rise to non-linear parametric coupling between the ESP field and the medium. The dynamical parameter $R_{e}$ is responsible for the electronic nonlinearity, and the dynamical parameter $R_{v}$ reflects electron-phonon nonlinearity. The phenomenological parameters $\alpha_{e, i}, \omega_{e, i}, T_{e, i}$ characterise the dispersion of the electronic and ionic linear polarisation responses. The coefficients $\beta$, $\gamma_{e, v}, \omega_{e 1, v}, T_{e 1, v}$ characterise the dispersion of the electronic and electronvibrational non-linear responses, respectively.

A sequence of the approximations (it has been detailed in [23]) allows to solve the equations (34) that leads to non-linear wave equation

$$
\frac{\partial E}{\partial z}-a \frac{\partial^{3} E}{\partial \tau^{3}}+b \int_{-\infty}^{\tau} E d \tau^{\prime}+g E^{2} \frac{\partial E}{\partial \tau}=\vartheta \Delta_{\perp} \int_{-\infty}^{\tau} E d \tau^{\prime}
$$

where $\tau=t-z / V$ is retarded time, $a, b, g$, and $\vartheta$ are positive parameters determined in [23]. $\Delta_{\perp}$ is the transverse Laplacian. The Ref. [24] represents the vector generation of the non-linear wave equation

$$
\frac{\partial \vec{E}}{\partial z}-a \frac{\partial^{3} \vec{E}}{\partial \tau^{3}}+b \int_{-\infty}^{\tau} \vec{E} d \tau^{\prime}+g(\vec{E} \cdot \vec{E}) \frac{\partial \vec{E}}{\partial \tau}+h \vec{E} \times\left(\vec{E} \times \frac{\partial \vec{E}}{\partial \tau}\right)=0
$$

describing propagation of the ESP of arbitrary polarisation. The numerical simulations shown that the polarisation self-action of ESP consists in a variation of the orientation of the vector of the electric field strength in the direction to the propagation axis proportional to the field squared and the velocity of rotation of its strength vector. There are no exact analytical results, unfortunately.

\subsection{Gas of quantum particles}

A self-consistent model for a non-linear interaction of an intense extremely short light pulse with a gas of atoms in framework of the quantum mechanics has been discussed in [25]. The model includes the scalar wave equation 
(14) and one-dimensional Schrödinger equation for an electron, which is subjected to the intratomic potential $U(x)$ and the optical radiation in the dipole approximation

$$
i \hbar \frac{\partial \psi}{\partial t}+\frac{\hbar^{2}}{2 m} \frac{\partial^{2} \psi}{\partial x^{2}}-U(x) \psi=e x E \psi
$$

The polarisation $P$ of the medium, which consists of model one-electron atoms with a number density $n_{A}$, is

$$
P(t)=-e n_{A} \int_{-\infty}^{+\infty} x|\psi|^{2} d x
$$

By using the Schrödinger equation we can rewrite the wave equation as

$$
\frac{\partial^{2} E}{\partial z^{2}}-\frac{1}{c^{2}} \frac{\partial^{2} E}{\partial t^{2}}=\frac{4 \pi e^{2} n_{A}}{c^{2} m} E+\frac{4 \pi e n_{A}}{c^{2} m} \int_{-\infty}^{+\infty}|\psi(x)|^{2} \frac{\partial U}{\partial x} d x
$$

The first term describes the linear response of the free electrons in the absence of the intratomic confining potential. The second term reflects the contribution of the coupled electrons. In 25] it was shown by numerical method that it is posible to generate a burst of harmonics with a relative power (in the region of 1\%) and a duration amounting toonly a few periods of the exciting optical field.

\subsection{Non-truncated Maxwell-Bloch model}

In order to investigate the self-induced transparency phenomena for extremely short electromagnetic pulses in [26] the non-truncated Maxwell-Bloch equations have been proposed. These equations take the form

$$
\begin{gathered}
\frac{\partial^{2} A}{\partial z^{2}}-\frac{1}{c^{2}} \frac{\partial^{2} A}{\partial t^{2}}=-\frac{4 \pi}{c} j-4 \pi k^{4} \chi_{3}|A|^{2} A, \\
\frac{\partial j}{\partial t}-i\left(\omega_{0}+\gamma_{s}|A|^{2}\right) j=i \frac{\left|m_{12}\right|^{2}}{\hbar c} \rho A, \\
\frac{\partial \rho}{\partial t}=\frac{2 i}{\hbar c}\left(j A^{*}-j^{*} A\right),
\end{gathered}
$$

where $A$ is a vector-potential of the electromagnetic wave, $j$ is atomic transition current density, $\rho$ is a population inverse density, $m_{12}$ is matrix element 
of resonant transition current. The Kerr and Stark effects were taken into account by introduction of the terms containing parameters $\chi_{3}$ and $\gamma_{s}$, respectively.

The results of the numerical simulations demonstrate the existence of the self-similar solutions of the system of equations (38). These solutions should be called by the solitons because they are stable in respect with pulse collisions. The main distinctive feature of the solitons of the non-truncated Maxwell-Bloch equations in that they do not break-up into the pulse sequence when the area of the incident pulse is higher then $2 \pi$.

\section{Conclusion}

We have considered some case of model media that allow the explicit temporal dependence of the electric field strength of the extremely short pulses to be determined by analytically. We did not make any assumptions concerning the harmonic carrier of the wave or the variation rate of the field in the pulse. As usually, only steady state solutions describing the propagation of the ESP can be found.

There are some investigations in the considered area that were left aside. However, it seems useful to mention works [27, 28, 29] where the propagation of a powerful electromagnetic pulses in a strongly non-linear medium without dispersion has been considered. The exactly solvable models are developed to describe the interaction of extremely short (few-cycle) transients with certain classes of insulators and conductors. Transient-excited fields are described analytically based on new, exact, non-periodic and non-stationary solutions to Maxwell's equations, obtained directly in time domain using a no Fourierexpansion, no time-space separation method. Such non-separable solutions form the mathematical basis of the non-periodic electromagnetic waves and make the standard harmonic wave concepts of frequency, phase, refraction index, and phase velocity irrelevant to the time description of non-periodic waves. Ref. [29] reviews these investigations.

\section{Acknowledgment}

We are grateful to Dr. S.V. Sazonov, Pr. S.A. Kozlov and Pr. A.V. Andreev

for valuable discussions. This work was supported by INTAS (European 
Union) under the grant No. 96-0339. 


\section{References}

[1] A.I. Maimistov, Quantum Electron. 30, (2000) 287.

[2] L. Allen, J.H. Eberly, Optical Resonance and Two-Level Atoms (Wiley, N.Y. 1975).

[3] R.K. Bullough, P.M. Jack, P.W. Kitchenside, R. Saudders , Phys.Scr. 20 (1979) 364.

[4] R.K. Bullough, F. Ahmad, Phys.Rev.Lett. 27 (1971) 330.

[5] S.L. McCall, E.L. Hahn, Phys.Rev.183 (1969) 457.

[6] S.V. Sazonov and E.V. Trifonov, J. Phys. B 27 (1994) L7.

[7] A.I. Maimistov, Quantum Electron. 27 (1997) 935.

[8] A.I. Maimistov, Optics and Spectrosc. 78 (1995) 435.

[9] A.E. Kaplan, Phys. Rev. Lett. 73 (1994) 1243; A. E. Kaplan, S.F. Straub and P. L. Shkolnikov, J.Opt.Soc.Amer. B 14, (1997) 3013.

[10] A.D. Vuzhva, Fiz.Tverd.Tela (Leningrad) 20 (1978) 272.

[11] A.I. Maimistov, Optics and Spectrosc. 87 (1999) 96

[12] L.D. Landau, E.M. Lifshits. Mechanics (Nauka Publishers: Moscow, 1973).

[13] S. Trillo, A.V. Buryak, Y.S. Kivshar, Opt. Comm. 122 (1996) 200.

[14] N. Bloembergen. Nonlinear Optics (Benjamin: New York, 1965).

[15] A. Yariv. Quantum Electronics (John Wiley and Sons, Inc.: New York and London, 1967).

[16] P.N. Butcher, D. Cotter. The Elements of Nonlinear Optics (Cambridge University Press, 1990).

[17] E.V. Kazantseva, A.I. Maimistov, Phys. Lett. A 263 (1999) 434.

[18] O. B. Dubrovskaya and A. P. Sukhorukov, Izv. RAN (Proceedings of the Russian Academy of Sciences), ser. Phys. 56 (1992) 184. 
[19] N.Bloembergen, Y.R. Shen, Phys.Rev.Lett. 12, (1964) 504.

[20] R.H. Pantell, H.E. Puthoff, Fundamentals of quantum electronics (John Wiley

[21] E.M. Belenov, P.G. Kryukov, A.V. Nazarkin, I.P. Prokopovich, Z.E.T.Ph. 105 (1094) 28 (in Russia)

[22] S.A. Kozlov, Optics and Spectrosc. 79 (1995) 267

[23] S.A. Kozlov, S.V. Sazonov, JETP 84 (1997) 221.

[24] S.A. Kozlov, Optics and Spectrosc. 84 (1998) 887.

[25] E.V. Vanin, A.V. Kim, A.M. Sergeev, M.C. Downer, JETP Lett. 58 (1993) 900.

[26] A.V. Andreev, Phys.Lett. A 179 (1993) 23; .A.V. Andreev, V.V. Berendakov, in ICONO'95: Coherent Phenomena and Amplification without Inversion, A.V. Andreev, O.Kocharovskaya, P. Mendel, Edts., Proceedings of SPIE, 2798, 112-120 (1996).

[27] A.B. Shvartsburg, L. Stenflo, P.K. Shukla, Phys.Rev. E 56 (1997) 7315

[28] A.B. Shvartsburg, Quantum Electron 26 (1998) 193.

[29] A.B. Shvartsburg, Usp.Fiz.Nauk 168 (1998) 85 (in Russia). 